\title{
Improve Immunity by Doing Yoga Asanas as Spiritual Healing A Review
}

\author{
Made Kurnia Widiastuti Giri ${ }^{1{ }^{1 *}}$ I Wayan Muderawan ${ }^{2}$ Komang Hendra Setiawan $^{1}$ \\ Ketut Indra Purnomo ${ }^{1}$ Putu Arya Nugraha ${ }^{1}$
}

\author{
${ }^{I}$ Medical Education Study Program, Universitas Pendidikan Ganesha, Singaraja, Indonesia \\ ${ }^{2}$ Chemistry Study Program, Universitas Pendidikan Ganesha, Singaraja, Indonesia \\ ${ }^{*}$ Corresponding author.Email: kurnia.widiastuti@undiksha.ac.id
}

\begin{abstract}
Today, citizens of the world are faced with enormous problems in various aspects of human life due to the problem of the COVID-19 pandemic. The COVID-19 pandemic has had a negative impact on all sectors of Indonesian people's lives. One of the sectors that was significantly affected was the health sector. The main factor that can prevent the transmission of the spread of COVID-19 that can hurt our bodies is by implementing a clean and healthy lifestyle and keeping our immune system stable and good. One way to maintain physical and mental health during the COVID-19 pandemic is to do spiritual healing which can have an impact in the form of a balanced mindset, mental resilience, and a strong immune system. One example of the application of spiritual healing is yoga. There are various types of yoga and one of them is yoga asanas. By doing yoga asanas, one can rejuvenate the body's skeletal framework, increase appetite, and this technique also helps in treating all digestive organs and can reduce mental tension or stress that can reduce the body's immune power (immunity) in dealing with microbes that interfere with our body. The movements in yoga asanas are Tadasana (Mountain Pose), Vrikshasana (Tree Pose), Adho Mukho Svanasana (Downward Dog Pose), Trikonasana (Triangle Pose), Kursiasana (Chair Pose), Naukasana (Boat Pose), Bhujangasana (Cobra Pose), Paschimottanasana (Forward Hunchback Pose), Balasana (Child Pose) and Sukhasana (Happy Pose). Yoga practice can activate the immune system by activating the thymus gland, which is located in the area between the heart and the breastbone. The thymus gland produces T-Cells which are a heterogeneous group of cells important in protecting the body against invasion by foreign organisms. With the various benefits described, the concept of spiritual healing, especially the application of yoga asanas, is very suitable to be applied during the COVID-19 pandemic in order to improve body and mental conditions for the better which will have an impact on increasing the body's immunity in preventing the entry of viruses or other antigens that can cause infection.
\end{abstract}

Keywords: COVID-19 Pandemic, Body Immunity, Physical and Mental Health, Spiritual Healing, Yoga Asanas.

\section{INTRODUCTION}

Starting in 2019, citizens of the world are faced enormous problems in various aspects of human life due to the COVID-19 pandemic. According to the World Health Organization (WHO), COVID-19 is a disease caused by the Severe Acute COPD (Chronic Ob Coronavirus 2 (SARS-CoV-2) causes the flu to more severe diseases including MERS-CoV and SARS-CoV. [1]. The increase in the number of COVID-19 cases took place quite quickly with the rate of infection increasing exponentially until it reached the Case Fatality Rate (CFR) of 2.3\%. Quoted from WHO data as of June 10, 2021 , there were $175,185,477$ confirmed cases of COVID-19 with deaths reaching 3,777,348. COVID-19 has spread to more than 185 countries, prompting the 
WHO to issue a statement regarding an international public health emergency and this disease is declared a pandemic disease. The COVID-19 pandemic has had a negative impact on all sectors of Indonesian people's lives, starting from the health, economic, tourism, social and other sectors [2]. One of the sectors that was significantly affected was the health sector. The health sector holds an important responsibility and at the same time faces serious problems in dealing with this case. The main factor that can prevent the transmission of the spread of COVID-19 that can hurt our bodies is by implementing a clean and healthy lifestyle and keeping our immune system stable and good. With this in mind, the government advises people to wear masks, wash their hands or maintain personal hygiene, and keep a distance in public places to prevent the transmission of the spread of COVID-19. In addition, the community can also increase immunity (immune power) in preventing the entry of the COVID-19 virus by maintaining mental and physical health. Community tips in maintaining mental and physical health including eating balanced nutritious foods, drinking enough water, getting enough sleep, exercising regularly, and keeping yourself from stress.

One way to relieve physical, mental, and mental fatigue is to do spiritual healing which can have an impact in the form of a balanced mindset, strong mental resilience, and strong body resistance. This can be clinically due to spiritual healing being able to balance the work of the glands and also relax the muscles. Likewise with the nervous system, stimulating circulation, and focusing the mind which causes the body and mind to be in harmony and one example of the application of spiritual healing is yoga.

Yoga is one way or activity that can provide benefits in eliminating physical, mental, and mental fatigue. This activity is one example of spiritual healing which is very popular in the world. There are various types of yoga and one of them is yoga asanas. Yoga asanas have an impact on every aspect of the human body, not only balancing the work of the glands but also relaxing the muscles, improving the nervous system, stimulating circulation, and focusing the mind. While performing these movements, the body remains in a relaxed efficient state and the deep breathing that accompanies these postures allows the blood to absorb more oxygen. During yoga asanas, more energy is collected than expended [3].

Some people have a misunderstanding about yoga asanas. Several perceptions state that yoga asanas are only physical exercises and have nothing to do with providing a path of spiritual awareness. This opinion is definitely wrong because although yoga asanas cannot provide spiritual awareness, yoga asanas are one of the stages on the spiritual path. Yoga asanas can strengthen the mind and are able to withstand pain. After completing yoga asanas, the practice of yoga asanas can provide benefits, namely the mind becomes balanced and the body's vitality is dynamic. In addition to the above benefits, there are other benefits that we can get by doing yoga asanas, namely that in doing asanas it takes good discipline and vigilance to get the benefits. Yoga asanas can be practiced by everyone, depending on age, health, and illness. Yoga asanas are the best method of maintaining and maintaining spinal flexibility and relieving spinal curvature. In addition, yoga asanas can also improve posture-related ailments, such as back pain, shoulder pain, joints, and stiffness.

Yoga asanas can also provide benefits in building, helping, repairing muscle tissue, improving the nervous system, eliminating fatigue, restoring lost energy, helping to keep the body and mind good [3].

This yoga asana method is very easy to do, the costs required are also relatively cheap, and it does not require a lot of material to do it because only a mat or carpet can do this yoga asana. Yoga asanas can provide new energy to the endocrine glands so that the body receives hormones that are useful for the continuity of body functions. By doing yoga asanas, one can rejuvenate the body's skeletal framework, increase appetite, and this technique also helps in treating all digestive organs and can reduce mental tension or stress that can reduce the body's immunity (immunity) in dealing with incoming viruses. With this in mind, the concept of spiritual healing, especially the application of yoga asanas, is very suitable to be applied during the COVID-19 pandemic in order to improve body and mental conditions for the better which will have an impact on increasing the body's immunity in preventing the entry of viruses or other antigens that can reduce the risk of infection.

\section{METHOD}

Google Scholar was used to search databases for yoga-related studies and therapies. Initially, the advanced search option was used to insert the phrases "yoga" and "therapeutic effects." This search was undertaken to gather broad information about yoga's therapeutic effects. A second search was run using the keywords or exact phrases "hatha yoga" and "yoga therapeutic effects". Insertion criteria are peer-reviewed articles published between 1990 and 2009; interventions using yoga and/or meditation; benefits of yoga on various outcomes were measured. Several steps were performed to choose the articles for this document. First, the title. The article was saved to a folder if it suited the study of yoga's therapeutic effects. We selected to focus on studies that described yoga-based therapies to improve health. The chosen papers were then read and reviewed in detail. The papers listed cover a wide range of yoga's applications and therapeutic effects. 


\section{RESULTS AND DISCUSSION}

\subsection{Definition of Spiritual Healing}

Spiritual healing is a technique or method that is useful in building physical, mental, and mental health through spiritual practices such as mantras, symbols, breathing energy, and meditation. In Bali, spiritual healing can be seen as tangible and intangible. Tangibly, it can be seen in terms of appearance, namely in the form of eco-spiritual healing that displays the uniqueness of the natural environment such as yoga. Cultural-spiritual healing performances show the uniqueness of rituals and cultural support for the local community. The performance of the spiritual healing eco-culture shows the uniqueness of the ashram tradition and the ashram environment. The appearance of the construction of spiritual healing eco-culture shows the uniqueness of the environment and culture that is the result of the construction. Intangible (not real), this uniqueness is built from the knowledge of healers and the experiences of foreign tourists. Knowledge of healers (medicine) can help to heal stress, awaken chakras, and connect foreign tourists with natural energy are the uniqueness of spiritual healing in Bali [4].

\subsection{Yoga Health Benefits for Several Diseases}

Depression, anxiety, stress, and insomnia are among the most common reasons people seek yoga therapy [5]. Yoga improves relaxation, slowing the breath and focusing on the present, balancing the sympathetic nervous system and the flight-or-fight reaction [6]. It lowers blood pressure, lowers cortisol levels, and promotes blood flow to the intestines and important organs.

Yoga aims to promote mental peace, relaxation, enhanced self-confidence, efficiency, increased concentration, decreased irritation, and a positive attitude on life [5]. Yoga creates balanced energy, which is essential for immune system function [5]. Yoga inhibits the hypothalamus's sympathetic region. Stress-related autonomic regulating reflex systems are restored as a result of this inhibition. Yoga activities reduce fear, aggression, and fury while increasing pleasure centres in the middle forebrain and other places. Yoga and meditation students have less anxiety, heart rate, respiration rate, blood pressure, and cardiac output [7][10].

Yoga relieves depression by increasing serotonin levels and decreasing monoamine oxidase, an enzyme that breaks down neurotransmitters and cortisol [6]. There are several ways to treat depression, but many people prefer alternative therapy because to side effects, lack of response, or just choice. Several studies show that yoga can help with sadness, tension, and anxiety [10][12].
Yoga's first and most noticeable effect is increased flexibility [6]. Yoga is supposed to help relieve aches and pains by gradually releasing the muscles and connective tissues surrounding the bones and joints. Yoga promotes muscle growth and strength, which protects against arthritis, osteoporosis, and back discomfort [6]. Yoga exercises stretch and soak joints, delivering fresh nutrients, oxygen, and blood to the area, preventing problems like arthritis and chronic pain [5]. Neglected cartilage will eventually wear away, revealing the underlying bone. Yoga, meditation, or a combination of the two has been demonstrated to relieve pain in persons with arthritis, back pain and other chronic diseases [5].

Yoga boosts blood flow and haemoglobin and red blood cell levels, allowing more oxygen to reach the body's cells [5]. Yoga thins the blood, reducing the risk of heart attack and stroke caused by blood clots. When released, twisting poses wring out venous blood from inside organs and allow oxygenated blood in. Inverted positions stimulate venous blood flow from the legs and pelvis back to the heart, where it is oxygenated. Yoga has been shown to reduce resting heart rate, increase endurance, and improve maximum oxygen intake and use [13]. Consistent aerobic exercise reduces the risk of heart attack [6]. While not all yoga is aerobic, even non-aerobic yoga movements can improve cardiovascular health.

\subsection{Benefits of Yoga Asanas During A Pandemic Covid 19}

At the time of the COVID-19 pandemic, many activities did not take place as before the pandemic because they were still under restrictions on community activities. This restriction makes some people who are still in a situation of not getting income from work even to the occurrence of layoffs (Termination of Employment) workers by the company which resulted in some people having to carry out their activities from home. This condition causes many people to experience boredom of mind, soul, and body. So one solution that we can do is practice yoga asanas which are used as a place to calm the human mind to get positive vibrations in every activity. Yoga asanas have many meanings broadly and transcend other physical practice practices as they not only have a physical effect on the muscles and bones, but also on one's mental health, soul and spiritual personality. All the faculties that exist in the mind, soul, and body can form a new energy system, a new spirit, and stimulate active attention. Yoga asanas can provide new energy to the endocrine glands so that the body receives hormones that are useful for the continuity of body functions. By doing yoga asanas, one can rejuvenate the body's skeletal framework, increase appetite, and this technique also helps in treating all digestive organs and can reduce mental tension or stress that can lower the body's immune system (immunity) in the face of incoming viruses. With this in mind, the concept of spiritual healing, especially 
the application of yoga asanas, is very suitable to be applied during the COVID-19 pandemic in order to improve body and mental conditions for the better which will have an impact on increasing the body's immunity in preventing the entry of viruses or other antigens that can reduce the risk of infection.

Therefore, yoga asanas are used as a physically and mentally healthy lifestyle during the new era normal for the COVID-19 pandemic. Yoga asanas are practiced with concentration, relaxation and calm without tension, and stimulate the nervous system and endocrine glands according to the functions of the human body.

\section{CONCLUSION}

Spiritual healing is a technique or method that is useful in building a healthy body, mind, and spirit through spiritual practices such as mantras, symbols, breathing energy, and meditation. Yoga is a continuous attempt to improve physical and mental wellness. The soul awareness in question is the awareness of one's authenticity which is carried out by the union of body, mind and soul, which of course is carried out with practical practices.

The types of yoga that develop in the community include ananda yoga, iyegar yoga, kundalini yoga, bikram yoga, ashtanga yoga, jivamukti yoga, sivananda yoga, kripalu yoga, meditation yoga, prenatal yoga, vini yoga, and acro yoga.

Yoga asanas are levels of yoga through postures. Comfortable body postures are called asanas. So, the main thing in yoga asanas are postures (forms of movement), pranayama (regulation of flowing breath), and bhanda (emphasis of breath on the area of breathing). Pranayama such as holding the breath against the chest, abdomen or diaphragm. Yoga asanas have an impact on every aspect of the human body, not only balancing the work of the glands but also relaxing the muscles, improving the nervous system, stimulating circulation, and focusing the mind.

By doing yoga asanas, one can rejuvenate the body's skeletal framework, increase appetite, and this technique also helps in treating all digestive organs and can reduce mental tension or stress that can reduce the body's immune power (immunity) in dealing with microbes that interfere with our body. The movements in yoga asanas are Tadasana (Mountain Pose), Vrikshasana (Tree Pose), Adho Mukho Svanasana (Downward Dog Pose), Trikonasana (Triangle Pose), Kursiasana (Seat Pose), Naukasana (Boat Pose), Bhujangasana (Cobra Pose), Paschimottanasana (Forward Hunchback Pose), Balasana (Child Pose) and Sukhasana (Happy Pose).

Yoga practice can activate the immune system by activating the thymus gland, which is located in the area between the heart and the breastbone. The thymus gland produces T-Cells, a heterogeneous group of cells important in protecting the body against invasion by foreign organisms. During the Pandemic, everyone experienced boredom of the mind, soul and body of the human being. So, one solution that we can do is practice yoga asanas which are used as a place to calm the human mind so that they get positive vibrations in every activity. Yoga asanas have many meanings broadly and transcend other physical practice practices as they not only have a physical effect on the muscles and bones, but also on one's mental health, soul and spiritual personality. Seeing the benefits provided by the implementation of yoga asanas which can be one of the supportive therapies in dealing with the COVID-19 pandemic. Some yoga asanas techniques that can be done standing posture to increase stamina, the body is more balanced and stable. Posture balance serves to make the muscles on both sides stable, good body coordination, focused mind, balanced left and right brain, improve posture. Sitting posture serves to increase body stability, concentration, reduce body fatigue, and increase body immunity. The sitting posture bent towards the front serves to unite the body and mind, strengthen the abdominal muscles, legs, spine. Posture opens the chest serves to strengthen the heart and reduce stress and anxiety. The hip joint flexing posture serves to release tension in the hip joint and prevent sciatica. Posture strengthens the arms and wrists serves to increase courage, concentration and body balance. Twist posture serves to refresh the central nervous system and detox toxins in the body. Inverted posture serves to relax and make the body more fit. Restorative posture serves to calm and balance in the body. With the various benefits described, the concept of spiritual healing, especially the application of yoga asanas, is very suitable to be applied during the COVID-19 pandemic in order to improve body and mental conditions to be better which will have an impact on increasing the body's immunity in preventing the entry of viruses or other antigens that can cause infection.

\section{REFERENCES}

[1] J. Zheng, "SARS-coV-2: An emerging coronavirus that causes a global threat," Int. J. Biol. Sci., vol. 16, no. 10, pp. 1678-1685, 2020, doi: $10.7150 /$ ijbs. 45053 .

[2] S. Syafrida and R. Hartati, "Bersama Melawan Virus Covid 19 di Indonesia," SALAM: Jurnal Sosial dan Budaya Syar-i, vol. 7, no. 6. pp. 495508, 2020, doi: 10.15408/sjsbs.v7i6.15325.

[3] I. G. M. W. Sena, "Kinesiologi Yoga Asanas (Kunci Kebahagiaan Tubuh, Pikiran Dan Jiwa)," Jurnal Yoga Dan Kesehatan, vol. 1, no. 1. p. 15, 2020, doi: 10.25078/jyk.v1i1.1539.

[4] I. G. Sutarya, "Spiritual Healing Dalam Pariwisata Bali: Analisis Tentang Keunikan, Pengembangan, Dan Kontribusi Dalam Pariwisata," Universitas Udayana, 2016. 
[5] K. Pilkington, G. Kirkwood, H. Rampes, and J. Richardson, "Yoga for depression: The research evidence," J. Affect. Disord., vol. 89, no. 1-3, pp. 13-24, 2005, doi: 10.1016/j.jad.2005.08.013.

[6] McCall T, "New York: Bantam Dell a Division of Random House Inc," Yoga as Medicine. 2007.

[7] J. R. Bharshankar, R. N. Bharshankar, V. N. Deshpande, S. B. Kaore, and G. B. Gosavi, "Effect of yoga on cardiovascular system in subjects above 40 years," Indian J. Physiol. Pharmacol., vol. 47, no. 2, pp. 202-206, 2003.

[8] M. Javnbakht, R. Hejazi Kenari, and M. Ghasemi, "Effects of yoga on depression and anxiety of women," Complement. Ther. Clin. Pract., vol. 15, no. 2, pp. 102-104, 2009, doi: 10.1016/j.ctcp.2009.01.003.

[9] D. A. Birkel and L. Edgren, "Hatha yoga: Improved vital capacity of college students," Altern. Ther. Health Med., vol. 6, no. 6, pp. 5563, 2000.

[10] A. Woolery, H. Myers, B. Sternlieb, and L. Zeltzer, "A yoga intervention for young adults with elevated symptoms of depression," Altern. Ther. Health Med., vol. 10, no. 2, pp. 60-63, 2004.

[11] S. Arora and J. Bhattacharjee, "Modulation of immune responses in stress by Yoga," Int. J. Yoga, vol. 1, no. 2, p. 45, 2008, doi: 10.4103/0973-6131.43541.

[12] A. Vedamurthachar et al., "Antidepressant efficacy and hormonal effects of Sudarshana Kriya Yoga (SKY) in alcohol dependent individuals," J. Affect. Disord., vol. 94, no. 1-3, pp. 249-253, 2006, doi: 10.1016/j.jad.2006.04.025.

[13] K. Harinath et al., "Effects of Hatha Yoga and Omkar Meditation on Cardiorespiratory Performance, Psychologic Profile, and Melatonin Secretion," J. Altern. Complement. Med., vol. 10, no. 2, pp. 261-268, 2004, doi: $10.1089 / 107555304323062257$. 